\title{
Obtención y caracterización de ferritas ternarias de manganeso por mecanosíntesis
}

\author{
Francisco Prieto-García ${ }^{1, *}$, Félix Sánchez de Jesús², María Aurora Méndez-Marzo², \\ Graciela García-Barrera ${ }^{3}$ y Alberto José Gordillo-Martínez ${ }^{1}$
}

${ }^{1}$ Centro de Investigaciones Químicas. Universidad Autónoma del Estado de Hidalgo, Carretera Pachuca-Tulancingo km 4.5, 42090, Ciudad Universitaria, Pachuca, Hidalgo, México.

${ }^{2}$ Centro de Investigaciones en Materiales y Metalurgia. Universidad Autónoma del Estado de Hidalgo, Carretera Pachuca-Tulancingo km 4.5, 42090, Ciudad Universitaria, Pachuca, Hidalgo, México.

${ }^{3}$ Centro de Estudios Académicos sobre Contaminación Ambiental. Universidad Autónoma de Querétaro, Cerro Las Campanas s/n. Santiago de Querétaro, México.

*prietog@uaeh.edu.mx

\begin{abstract}
Resumen
Partículas cristalinas de $\mathrm{MnFe}_{2} \mathrm{O}_{4}$ fueron sintetizadas por molienda y mezclado en un molino de bola, obteniéndose a partir de mezcla estequiométrica de manganosita $(\mathrm{MnO})$ y hematite $\left(\alpha-\mathrm{Fe}_{2} \mathrm{O}_{3}\right)$. El proceso de mecanosíntesis fue realizado a temperatura ambiente en recipientes de acero endurecido y con frascos del carburo de tungsteno. El análisis cuantitativo de las fases se realizó por difracción de rayos X del polvo. El método de Rietveld fue utilizado para estudiar las transformaciones químicas producidas por la acción de la molienda de los polvos.

La fase de espinela del compuesto cristalino $\mathrm{MnFe}_{2} \mathrm{O}_{4}$ comienza a aparecer después de $10 \mathrm{~h}$ de molienda y alcanza su contenido máximo (fracción molar aproximadamente 0.8 ) después de $35 \mathrm{~h}$ de molienda. Una prolongada molienda indujo a una contaminación severa en la mezcla del polvo con hierro metálico cuando se utilizó el recipiente de acero inoxidable endurecido. La contaminación con Fe se origina en el interior del recipiente por el deterioro de las bolas. La fricción de las bolas puede inducir una reacción redox entre $\mathrm{Fe}(\mathrm{III})$ y el hierro metálico, transformando la fase de la espinela sintética $\mathrm{MnFe}_{2} \mathrm{O}_{4}$ en una fase del tipo wustita (Fe, Mn)O. La permeabilidad magnética a los diferentes tiempos de molienda lo demuestra.
\end{abstract}

Palabras clave: Ferrita del manganeso; Mecanosíntesis; Ferrita cristalina; Permeabilidad magnética; Método de Rietveld.

Abstract

Crystalline $\mathrm{MnFe}_{2} \mathrm{O}_{4}$ particles were synthesized by a high-energy ball milling technique, starting from a manganosite (MnO) and hematite $\left(\alpha-\mathrm{Fe}_{2} \mathrm{O}_{3}\right)$ stoichiometric powder mixture. The mechanosynthesis process was performed at room temperature both in hardened steel and in tungsten carbide vials. X-ray powder diffraction quantitative phase analysis by the Rietveld method was used to study the chemical transformations promoted by the milling action. The crystalline $\mathrm{MnFe}_{2} \mathrm{O}_{4}$ spinel phase begins to appear after $10 \mathrm{~h}$ of milling and reaches its maximum content ( $\approx 0.8$ molar fraction) after $35 \mathrm{~h}$ of milling. A prolonged milling time induces a dramatic contamination of the powder mixture, when hardened stainless steel was adopted, due to metallic iron originating from vial and balls debris. Ball milling is able to induce a redox reaction between FeIII and metallic iron, transforming the MnFe2O4 spinel phase into a wüstite type ( $\mathrm{Fe}, \mathrm{Mn}) \mathrm{O}$ phase. Magnetic permeability in different time of miller demonstrates.

Key words: Manganese ferrite; Mechanosynthesis; Nanostructured ferrite; Magnetic permeability; Rietveld method. 


\section{Introducción}

Las ferritas representan unos de los materiales más ampliamente estudiados por sus características eléctricas y magnéticas. Estos materiales se utilizan en la industria eléctrica y electrónica para la fabricación de dispositivos y de componentes tales como centro magnético de alta densidad de los cabezales de lectura y grabación. Además del uso tradicional, un interés renovado ahora está emergiendo en diversos campos tales como ciencia biomédica (Saferikova y Safarik, 2001; Bergey et al., 2002) o en investigaciones en materias energéticas sostenibles.

Se ha probado que algunas ferritas se pueden utilizar con éxito en el agua a baja temperatura en los ciclos termoquímicos (Tamaura et al., 1995; Tamaura et al., 1999).

Una mezcla de ferrita de manganeso y de carbonato de sodio reacciona entre $800-1000{ }^{\circ} \mathrm{C}$ produciendo ferrita cuaternaria de sodio y manganeso, $\mathrm{CO}_{2}$ e hidrógeno, siguiendo la reacción que se propone (1):

$$
\begin{array}{rl}
2 \mathrm{MnFe}_{2} \mathrm{O}_{4}(\mathrm{~s})+ & 3 \mathrm{Na}_{2} \mathrm{CO}_{3}(\mathrm{~s})+\mathrm{H}_{2} \mathrm{O}= \\
6 & \mathrm{Na}\left(\mathrm{Mn}_{1 / 3} \mathrm{Fe}_{2 / 3}\right) \mathrm{O}_{2}(\mathrm{~s})+3 \mathrm{CO}_{2}(\mathrm{~g})+\mathrm{H}_{2}
\end{array}
$$

al finalizar la producción de hidrógeno, los reactivos iniciales se pueden regenerar a $600^{\circ} \mathrm{C}$, cerrando el ciclo según la reacción (2)

$$
\begin{aligned}
& 6 \mathrm{Na}\left(\mathrm{Mn}_{1 / 3} \mathrm{Fe}_{2 / 3}\right) \mathrm{O}_{2}(\mathrm{~s})+3 \mathrm{CO}_{2}(\mathrm{~g})= \\
& 2 \mathrm{MnFe}_{2} \mathrm{O}_{4}(\mathrm{~s})+3 \mathrm{Na}_{2} \mathrm{CO}_{3}(\mathrm{~s})+0.5 \mathrm{O}_{2}
\end{aligned}
$$

En el primer paso, la ferrita de $\mathrm{Mn}(\mathrm{II})$ es oxidada por la presencia del agua a $\mathrm{Na}\left(\mathrm{MnIII}{ }_{1 / 3} \mathrm{Fe}_{2 / 3}\right) \mathrm{O}_{2}$ y el hidrógeno se produce junto con el bióxido de carbono. Entonces, el ion sodio mezclado en los óxidos de manganeso y hierro, reacciona con bióxido de carbono; durante el proceso se desprende oxígeno y la ferrita del manganeso se regenera. Varios métodos sintéticos han sido desarrollados para obtener polvos de ferrita. Los ejemplos significativos son las reacciones de estado sólido de alta temperatura (Tamaura et al., 1998; Kaneko et al., 2002) de precursores del óxido o de los hidróxidos coprecipitados (Kodama et al., 1996), de los oxalatos (Bremer et al., 1992; Guillot et al., 1997), de los citratos (Gajbhiye y Balaji, 2002), así como los procesos que promueven la precipitación directa de la fase espinela sintética de la micela y de los sistemas reversos de la micela (Carpenter et al., 1999; Liu et al., 2000; Sun et al., 2004).

Recientemente ha sido demostrada la eficacia de la técnica por molienda en molinos de bola de gran energía, para la obtención de ferritas nanoestructuradas por mecanosíntesis y por la activación mecánica del $\mathrm{MnO}$ y su mezcla con $\alpha-\mathrm{Fe}_{2} \mathrm{O}_{3}$ (Albani et al., en prensa), según la reacción (3).

$$
\mathrm{MnO}+\alpha-\mathrm{Fe}_{2} \mathrm{O}_{3}=\mathrm{MnFe}_{2} \mathrm{O}_{4}
$$

Cuando la ferrita de manganeso está mezclada con carbonato de sodio, se puede producir la reducción del agua, según lo descrito en la reacción (1) a $700^{\circ} \mathrm{C}$, un valor de temperatura más bajo que lo obtenido para el reactivo microcristalino obtenido de forma tradicional (Tamaura et al., 1995). A pesar de ese resultado positivo, la mezcla nanocristalina obtenida presenta una eficiencia de reacción cercana al 60\% (Albani et al., en prensa). Este resultado se relaciona con la difusión del oxígeno que se produce dentro del frasco durante el proceso de mecanosíntesis, causando una oxidación parcial de Mn(II) a Mn(III). Por consiguiente, solamente una fracción del manganeso contenido en el polvo sigue siendo capaz de producir el hidrógeno del agua.

Con la finalidad de obtener una ferrita de manganeso lo más pura posible, la mecanosíntesis del compuesto fue realizada bajo condiciones experimentales controladas de modo que la difusión del oxígeno en los frascos se evitara. El proceso en estado sólido de la síntesis fue supervisado cuantitativamente usando difracción de rayos $\mathrm{X}$ de polvo (DRX) para las muestras que se obtienen a diferentes tiempos de molienda y aplicando un análisis cuantitativo de la fase por el método de Rietveld en los espectros de DRX. Un método similar fue aplicado recientemente en otras ferritas nanocristalinas en los trabajos de Bid y Pradhan (2003; 2004).

Las muestras seleccionadas fueron probadas para evaluar la eficacia de las ferritas obtenidas en la reacción de producción de hidrógeno.

\section{Metodología experimental}

\subsection{Proceso de molienda}

El proceso de mecanosíntesis por molienda se realizó usando un molino planetario de bolas (Fritsch Pulverisette 5), equipado con frascos cilíndricos de acero endurecido $\left(250 \mathrm{~cm}^{3}\right)$ y con enfriamiento por aire comprimido. Para evitar los fenómenos oxidativos asociados a la difusión del oxígeno, las tapas estándares de los frascos fueron substituidas por tapas especiales, equipadas con una válvula hermética en las juntas y un anillo en la base. De esta manera, los frascos podían mantener un nivel de vacío de 1 mbar durante el tiempo de molienda. La síntesis fue realizada llenando el frasco con $10 \mathrm{~g}$ de mezcla estequiométrica de $\alpha-\mathrm{Fe}_{2} \mathrm{O}_{3}$ y $\mathrm{MnO}$ (en ambos casos, Aldrich, con pureza de 99\%). Las bolas de acero inoxidables fueron de $15 \mathrm{~mm}$ de diámetro y una relación peso de polvo/bola de 1/10. El vacío del sistema de 1 mbar permite estimar que el contenido en oxígeno es de alrededor 0.2 mbar. La velocidad de rotación fue de $320 \mathrm{rpm}$. Para prevenir un calentamiento excesivo de los frascos, los experimentos fueron realizados alternando 10 minutos de molienda con 10 minutos de reposo (Magini et al., 1993). 


\subsection{Análisis por Difracción de rayos X (DRX)}

La efectividad de la mecanosíntesis y su evolución fue evaluada por DRX en un difractómetro PHILIPS, modelo PW-1710-BASED, con fuente de radiación $\mathrm{CuK} \alpha, \lambda=$ $0,15406 \mathrm{~nm}$, filtro de níquel, portamuestra de Aluminio, tensión del generador y corriente de $40 \mathrm{KV}$ y $30 \mathrm{~mA}$, respectivamente, con barridos de ángulos (20) de $5 \mathrm{a} 40^{\circ}$ a un tamaño de paso de $0.02^{\circ}$ en un tiempo de 30 segundos. La identificación cualitativa de las fases fue realizada por comparación, usando la base de datos PDF-2 (PCPDF-WIN, 1998). El análisis cuantitativo fue realizado sobre la base de los refinamientos del método propuesto por Rietveld (Rietveld, 1967; Young, 1993), utilizando el software de MAUD (Lutterotti et al., 2003) y la base de datos de ICSD (FIZ Karlsruhe, 1999).

El análisis cuantitativo de las fases de los sólidos que fueron obtenidos durante las transformaciones químicas y a diferentes tiempos de molienda, fue realizado por aplicación del método de Rietveld (Madsen et al., 2001). Usando una muestra molida por $10 \mathrm{~h}$, los parámetros de la celda unidad y otros datos cristalográficos, fueron refinados y relacionados con ambos productos y reactivos. Los resultados del refinamiento así obtenidos, fueron utilizados para el modelo de la muestra molida por $25 \mathrm{~h}$ y los resultados de esta muestra fueron aplicados a la muestra molida por $35 \mathrm{~h}$ y todas las muestras restantes, fijando en estos casos los parámetros de la celda para las fases que aparecieron durante la reacción de mecanosíntesis (reacción 3).

\subsection{Análisis químico}

Los contenidos de hierro y manganeso en las muestras sintetizadas fueron medidos por análisis espectrofotométrico de absorción atómica (EAA) en un equipo Perkin Elmer, modelo ANALYST 100, con llama acetileno-aire, flujo de aire de $0.8-1.1 \mathrm{~L} / \mathrm{min}$ y flujo de acetileno de 0.8 a $1.2 \mathrm{~L} / \mathrm{min}$. Se realizó una curva de calibrado con disolución patrón y mediante el programa FIT-2 con ajuste de regresión lineal obteniéndose valores de $\mathrm{r}^{2}=0.9981 \mathrm{y} \mathrm{r}^{2}=0.9916$ para el caso del hierro y manganeso, respectivamente.

\subsection{Análisis de permeabilidad magnética} de las ferritas

A los polvos de las ferritas sintetizadas a diferentes tiempos de molienda se les evaluó la permeabilidad magnética. Con ayuda de una bobina fabricada para tales propósitos; 100 vueltas de hilo de cobre, con $31.7 \mathrm{~mm}$ de largo y $16.7 \mathrm{~mm}$ de ancho. Este dispositivo permite medir el voltaje inducido que es directamente proporcional al valor de la inducción magnética provocada por la muestra (L). Después de medir la inducción del voltaje en la celda vacía $\left(\mathrm{L}_{0}\right)$, los polvos se colocaron en tubos de ensayos de vidrio de $100 \mathrm{~mm}$ de largo y $8 \mathrm{~mm}$ de diámetro interior, se compactaron adecuadamente y se taparon con algodón. Se colocaron en el interior de la bobina cada uno de los tubos conteniendo las muestras y se medió la inducción magnética provocada por la muestra (L). Los resultados de las mediciones se procesaron de usando la formula siguiente:

$$
\mu_{\text {rel }}=1+\mathrm{C}\left(\mathrm{L}-\mathrm{L}_{0}\right) \cdot 10^{4} / \mathrm{n} \cdot \pi \cdot \mathrm{b}^{2}
$$

donde:
$\mu_{\mathrm{rel}}:$ Permeabilidad magnética relativa
L: Inducción magnética en la muestra, $\mu \mathrm{H}$
$\mathrm{L}_{0}$ : Inducción magnética en el vacío, $\mu \mathrm{H}$
n: Número de vueltas del hilo de cobre en la bobina
C: Largo del enrollado en la bobina
b: Diámetro interior de los tubos de vidrio donde se colocó la muestra.

La susceptibilidad magnética $(\chi)$ se determinó a partir de la permeabilidad magnética relativa, según la expresión:

$$
\mu_{\text {rel }}=1+4 \pi \chi_{\text {rel }}
$$

Los valores de la susceptibilidad magnética relativa de las sólidos (ferritas) obtenidos se compararon con las de una magnetita pura $\left(\mathrm{Fe}_{3} \mathrm{O}_{4}\right)$, cuyo valor (adimensional) es 10 veces superior a la del vacío y se expresará en tanto por ciento según:

$$
\% \chi=\chi_{\text {rel }} / 10 \cdot 100=\chi_{\text {rel }} \cdot 10
$$

\subsection{Análisis de tamaño de partículas}

Las ferritas sintetizadas fueron evaluadas en un analizador de tamaño de partículas por rayos Laser LS13-320 de la marca Beckman Coulter. Muchas tecnologías utilizan la dispersión de luz para tener información sobre los materiales. Entre estas tecnologías, la luz de dispersión elástica (ELS) es el principal método utilizado para la caracterización de tamaños de partículas en un rango de micrones o milímetros. En ELS la luz dispersada tiene la misma frecuencia que la luz incidente, y la intensidad de la luz dispersada es una función de las propiedades ópticas de las partículas y sus dimensiones (Beckman Coulter, 2001). Se trabajaron en suspensión con agua con el módulo para suspensiones líquidas.

\section{Resultados y discusión}

\subsection{Identificación de fases}

La Figura 1 muestra los espectros de DRX de los polvos de ferritas sintetizadas, secuenciadas en base a los distintos tiempos de molienda. Se puede observar que a tiempo $0 \mathrm{~h}$, inicio de los experimentos, solo se presentan mezcla de las 




Figura 1. Difractogramas de las ferritas sintetizadas por mecanosíntesis a diferentes tiempos de molienda y a partir de una mezcla estequiométrica de $\mathrm{MnO}-\mathrm{Fe}_{2} \mathrm{O}_{3}\left(1: 1 \%\right.$ mol). Fases: 1) $\left.\left.\left.\mathrm{Fe}_{2} \mathrm{O}_{3}, 2\right) \mathrm{MnO}, 3\right) \mathrm{MnFe}_{2} \mathrm{O}_{4}, 4\right)$ $(\mathrm{FeO})_{x}(\mathrm{MnO})_{1-x}$ y 5$) \mathrm{Fe}$.

fases de $\alpha-\mathrm{Fe}_{2} \mathrm{O}_{3}$ [carta de difracción 33-664] y $\mathrm{MnO}$ [carta de difracción 7-230]. A partir de las $10 \mathrm{~h}$ de molienda la fase principal que se aprecia corresponde a la espinela cúbica de $\mathrm{MnFe}_{2} \mathrm{O}_{4}$ [carta de difracción 10-319], ya mayoritaria, aunque con la presencia de las fases anteriores. A partir de este tiempo las fases anteriores van desapareciendo hasta que a las 35 h sólo se observa la fase de la ferrita de manganeso estequiométrica.

A partir de $50 \mathrm{~h}$ de molienda, se aprecia una nueva fase identificada como $(\mathrm{FeO})_{x}(\mathrm{MnO})_{1-x}$ así como la fase de Fe metálico. Simultáneamente a la aparición de estas impureza, disminuye la fase $\mathrm{MnFe}_{2} \mathrm{O}_{4}$ hasta que a $85 \mathrm{~h}$ de molienda desaparece totalmente y prevalece como una única fase $(\mathrm{FeO})_{x}(\mathrm{MnO})_{1-x}$.

En un trabajo reportado [14], los autores identificaron una ferrita del tipo cuaternaria $\left(\mathrm{MnII}_{0.39} \mathrm{FeIII}{ }_{0.61}\right)\left[\mathrm{MnII}_{0.31}\right.$ $\mathrm{MnIII}_{0.53} \mathrm{FeIII}_{1.15} \mathrm{O}_{4.04}$, con la presencia de Mn(II) y Mn(III) y la composición obtenida fue asociada con la contaminación por oxígeno durante el proceso de molienda. En este trabajo, se aplicó un control cuidadoso del sistema que ha permitido evitar la contaminación por oxígeno en los frascos de reacción, por los cual no es atribuible o justificable la presencia de $\mathrm{Mn}$ (III). Después de $50 \mathrm{~h}$ de molienda, el $\mathrm{MnO}$ y el $\alpha-\mathrm{Fe}_{2} \mathrm{O}_{3}$ ya han desaparecido totalmente. En el mismo tiempo, nuevos picos aparecieron y se corresponden al pico principal del Fe metálico [carta de difracción 6-696]. Los picos bien definidos que se observan en $16.5,18.7$ y $26.6^{\circ}$ del ángulo $2 \theta$, pueden ser asignados a un óxido mezclado de manganeso y hierro que pudiera tener una estructura del tipo wustita [carta de difracción 77-2356]. Su contenido aumenta con el tiempo y después de $85 \mathrm{~h}$ de molienda, se convierte en la única especie distinguible.

El proceso de molienda fue realizado a presión de 0.2 mbar de $\mathrm{O}_{2}$. A esta presión, la fase del mangano-wustita puede coexistir con la jacobsita solamente a temperaturas superiores a los $1450^{\circ} \mathrm{C}$ según se ha reportado (Bonsdorf et al., 1998). Durante la molienda, la temperatura en los frascos fue menor de $60^{\circ} \mathrm{C}$ así mismo, el aumento puntual atribuible a la fricción de choques entre las bolas no debió exceder en $5^{\circ} \mathrm{C}$, valor calculado por aproximación adiabática del volumen estimado y según proponen otros autores (Maurice y Courtney, 1990; Magini e Iasonna, 1995), considerando el material en polvo, como material cerámico.

Durante la mecanosíntesis, la temperatura es baja para asumir que pueda ocurrir la descomposición de la jacobsita $\left(\mathrm{MnFe}_{2} \mathrm{O}_{4}\right)$ por reducción de $\mathrm{Fe}(\mathrm{III})$ en presencia de oxígeno. Por los resultados de los análisis de absorción atómica, se pudo observar un aumento significativo de la relación molar de Fe/Mn (hasta 2.6) hasta las $50 \mathrm{~h}$ de molienda, según se muestra en la Tabla 1. Este resultado indica la existencia de una contaminación notable por el hierro proveniente de las bolas de acero, que empieza a desaparecer a partir de las 70 horas.

\subsection{Análisis cuantitativo de fases}

La Figura 2 muestra el análisis de las fases, como resultado del proceso del refinamiento en la muestra molida para $25 \mathrm{~h}$ (se muestra como ejemplo representativo del refinamiento realizado a las muestras por el método Rietveld).

En la Tabla 2, se muestran resultados de dicho proceso

Tabla 1. Resultados de análisis por absorción atómica de Fe y Mn a diferentes tiempos de molienda en horas $(\mathrm{h})$, relación $[\mathrm{Fe}] /[\mathrm{Mn}]$.

\begin{tabular}{cccc}
\hline mg Mn/g & mg Fe/g & {$[\mathbf{F e}] /[\mathbf{M n}]$} & $\begin{array}{c}\text { Tiempo } \\
\text { molienda (h) }\end{array}$ \\
\hline 42.84 & 66.92 & 1.56 & 0 \\
58.64 & 64.02 & 1.09 & 10 \\
57.66 & 65.16 & 1.13 & 25 \\
42.14 & 77.54 & 1.84 & 35 \\
30.88 & 79.97 & 2.59 & 50 \\
41.44 & 76.04 & 1.83 & 70 \\
59.94 & 64.14 & 1.07 & 85 \\
\hline
\end{tabular}




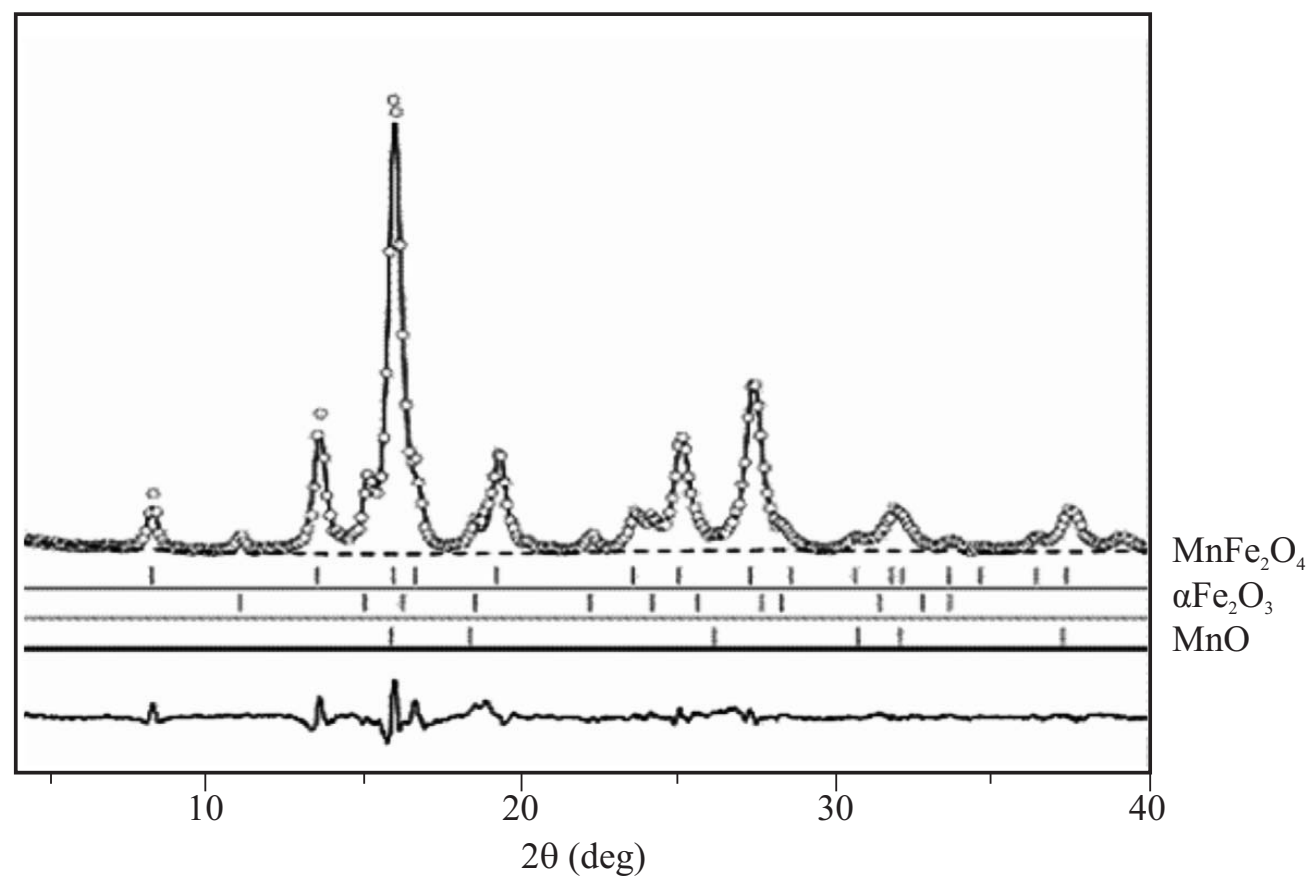

Figura 2. Señalados con (o) los patrones de difracción experimentales, señalados con (-) los calculados para la mezcla $\mathrm{MnO}-\mathrm{Fe}_{2} \mathrm{O}_{3}$ molida por $25 \mathrm{~h}$ en frasco de acero endurecido. La línea de abajo representa la diferencia entre los datos calculados y experimentales.

de refinamiento. Las fracciones molares de las fases de polvo obtenidas del análisis por el método de Rietveld en función del tiempo de molienda (ver Figura 3), indica que la fracción del jacobsita aumenta a expensas de la hematites y de la manganosita en las etapas de molienda tempranas, alcanzando su valor máximo (79\% mol), después de $35 \mathrm{~h}$ de molienda. Después de $50 \mathrm{~h}$, el contenido de la fase de espinela sintética disminuye por debajo de $50 \%$ mol y en el mismo tiempo, una cantidad considerable de hierro metálico está presente en la mezcla del polvo $(16 \% \mathrm{~mol})$.

El hierro metálico que se origina por la fricción y choque entre las bolas y con el interior del frasco de molienda, es detectable a partir de las $35 \mathrm{~h}$ de molienda llegando a ser relevante a las $50 \mathrm{~h}$, debido a la acción de la molienda prolongada de grandes cantidades de la espinela de Mn$\mathrm{Fe}_{2} \mathrm{O}_{4}$, al parecer propician una erosión apreciable de las piezas (bolas). Cuando el hierro alcanza un alto nivel de contaminación, pueden ocurrir reacciones químicas indeseadas en las interfaces de las partículas de $\mathrm{Fe} / \mathrm{MnFe}_{2} \mathrm{O}_{4}$. Significativos son los resultados obtenidos del análisis de la muestra molida para $85 \mathrm{~h}$. Según lo demostrado en el patrón de DRX (Figura 1), solamente la fase tipo wustita es, al parecer perceptible, mientras que el análisis cuantitativo aplicando el método de Rietveld, indica la presencia de una cantidad considerable de jacobsita no reactiva (aproximadamente el $40 \% \mathrm{~mol})$.

La fase de jacobsita desaparece debido a una descomposición progresiva que ocurre en los interfaces de Fe/ferrita. El hierro metálico en presencia de la ferrita aparentemente propicia un proceso mecanoquímico; después de $50 \mathrm{~h}$ de molienda, la fase ferrita es predominante $(99.5 \%$ peso) y la relación molar $\mathrm{Fe} / \mathrm{Mn}$ aumenta sensiblemente.

Tabla 2. Parámetros principales para la red, obtenidos del análisis de DRX y el método Rietveld para $\mathrm{MnO}, \mathrm{Fe}_{2} \mathrm{O}_{3}$, $\mathrm{MnFe}_{2} \mathrm{O}_{4}, \mathrm{Mn}_{\mathrm{x}} \mathrm{Fe}_{1-\mathrm{x}} \mathrm{O}$ y Fe. Tiempo de molienda en horas (h), relación Fe/Mn.

\begin{tabular}{lcccccc}
\hline $\begin{array}{l}\text { Tiempo de } \\
\text { molienda (h) }\end{array}$ & $\begin{array}{c}\mathrm{Fe}_{2} \mathrm{O}_{3} \\
\mathrm{a}(\mathrm{nm})\end{array}$ & $\begin{array}{c}\mathrm{MnO} \\
\mathrm{a}(\mathrm{nm})\end{array}$ & $\begin{array}{c}\mathrm{MnFe}_{2} \mathrm{O}_{4} \\
\mathrm{a}(\mathrm{nm})\end{array}$ & $\begin{array}{c}\mathrm{Mn}_{\mathrm{x}} \mathrm{Fe}_{1-\mathrm{x}} \mathrm{O} \\
\mathrm{a}(\mathrm{nm})\end{array}$ & $\begin{array}{c}\mathrm{Fe} \\
\mathrm{a}(\mathrm{nm})\end{array}$ & $\begin{array}{c}\text { Relacion Fe/Mn } \\
\text { calculada }\end{array}$ \\
\hline 0 & 0.503 & 0.445 & & & & 2.04 \\
10 & 0.503 & 0.445 & 0.846 & & & 2.04 \\
25 & 0.503 & 0.445 & 0.846 & & & 2.04 \\
35 & 0.503 & 0.445 & 0.846 & & 0.287 & 2.04 \\
50 & 0.503 & & 0.846 & 0.438 & 0.287 & 2.83 \\
70 & & & 0.846 & 0.438 & 0.287 & 2.77 \\
85 & & 0.846 & 0.438 & 0.287 & 2.96 \\
\hline
\end{tabular}




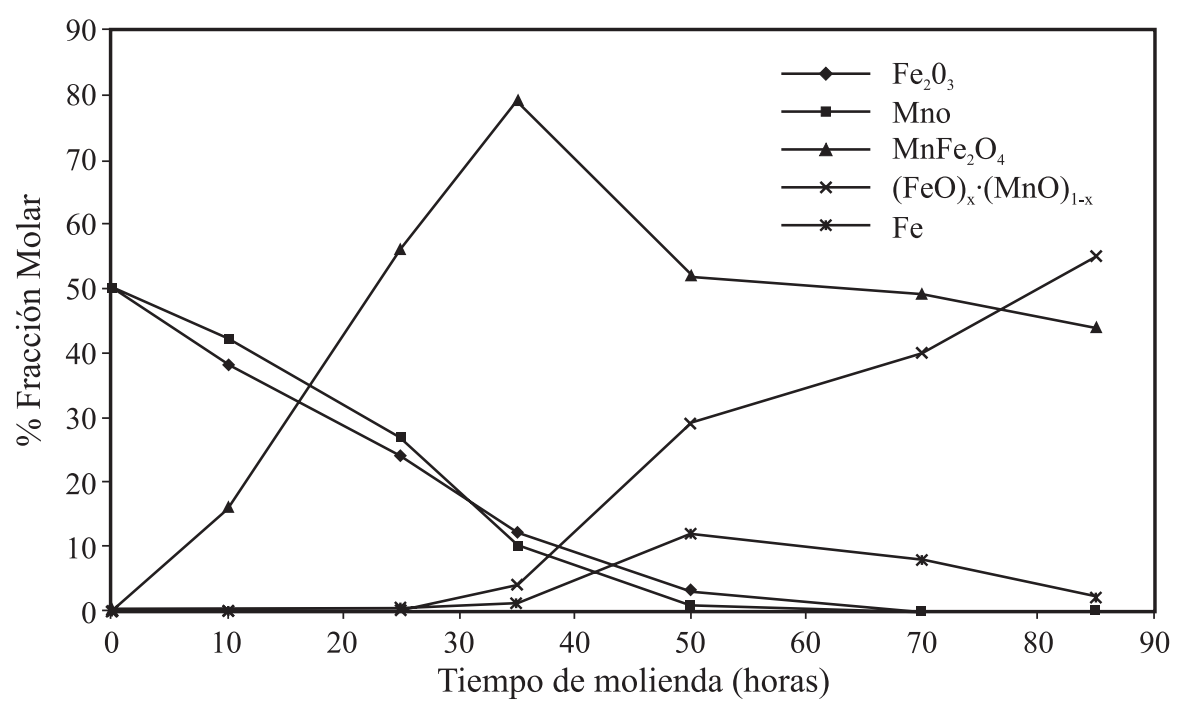

Figura 3. Variación de la fracción molar (mol \%) de las mezclas contra tiempo de molienda.

\subsection{Análisis de la Permeabilidad Magnética}

Los resultados de las evaluaciones de permeabilidad magnética de las ferritas obtenidas se observan en la Figura 4. Se aprecia una sensible permeabilidad magnética que inicia un ascenso en la medida en que comienza la aparición de la fase $\mathrm{MnFe}_{2} \mathrm{O}_{4}$ a partir de las $10 \mathrm{~h}$ de molienda, aunque mucho más baja que para una magnetita pura $(96.0 \mathrm{kHz})$. Destacan en dicha Figura los valores correspondientes a tiempo de molienda de $35 \mathrm{~h}$, con valores mayores de $80 \mathrm{kHz}$ (83.3), coincidiendo con los resultados de mayor porcentaje de fracción molar de $\mathrm{MnFe}_{2} \mathrm{O}_{4}$ obtenida, lo que permite corroborar que el tiempo de $35 \mathrm{~h}$ de molienda puede ser considerado como tiempo óptimo para el proceso que rinde la mayor proporción de ferrita estequiométrica de $\mathrm{Mn}$ (II). Este resultado se corresponde gráficamente con la Figura 3 para la aparición y desaparición de la $\mathrm{MnFe}_{2} \mathrm{O}_{4}$.

Según las series experimentales se lograron obtener valores de permeabilidades magnéticas para las ferritas de manganeso desde 39.0 hasta $83.3 \mathrm{kHz}$. Estos resultados permiten compararlas con la magnetita $\left(\mathrm{Fe}_{3} \mathrm{O}_{4}\right)$ con un valor de permeabilidad magnética de $96.0 \mathrm{kHz}$, esto se corresponde desde un $40.6 \%$ hasta un $86.8 \%$ de similitud en esta propiedad.

\subsection{Análisis del tamaño de partículas}

Los tamaños de partículas de los sólidos que se han obtenido, se pueden apreciar en la Tabla 3, donde se muestra una gran variación con el tiempo de molienda. Se puede observar que en el inicio del proceso los tamaños promedios corresponden a los esperados para la mezcla de $\mathrm{MnO}$ y $\alpha-\mathrm{Fe}_{2} \mathrm{O}_{3}$. A partir de las $10 \mathrm{~h}$ de molienda se observó un incremento del tamaño promedio de las partículas que debe estar asociado a la presencia de la fase de la espinela cúbica de $\mathrm{MnFe}_{2} \mathrm{O}_{4}$ que empieza a aparecer. En la medida en que desaparece la fase de $\mathrm{MnO}$ principalmente, las dimensiones promedio de las partículas disminuyen hasta llegar a las $35 \mathrm{~h}$ de molienda donde se alcanza el menor tamaño promedio así como la menor variabilidad de éstos (mejor homogeneidad en general). Posteriormente y con la aparición de otras fases como $(\mathrm{FeO})_{x}(\mathrm{MnO})_{1-x}$ y la fase de $\mathrm{Fe}$, el tamaño promedio de nueva cuenta muestra un incremento, así como mayores variabilidades.

\section{Conclusiones}

Se ha demostrado la posibilidad del proceso de obtención de una ferrita estequiométrica de manganeso por la vía de mecanosíntesis. En este proceso en estado sólido, se pudo evaluar la composición de la mezcla sólida, con auxilio de la difracción de rayos $\mathrm{X}$ y usando el análisis

Tabla 3. Tamaños de partículas de las mezclas sintetizadas a diferentes tiempos. Se pueden observar los tamaños promedios y los valores de partículas menores de $0.45 \mu \mathrm{m}$ así como la variabilidad.

\begin{tabular}{|c|c|c|c|c|}
\hline \multirow[t]{2}{*}{$\begin{array}{l}\text { Tiempo de } \\
\text { molienda }(\mathrm{h})\end{array}$} & \multicolumn{2}{|c|}{$\begin{array}{c}\text { Tamaños de Partículas } \\
(\mu \mathrm{m})\end{array}$} & \multicolumn{2}{|c|}{$\begin{array}{l}\text { Variabilidad de tamaños } \\
\qquad(\mu \mathrm{m})\end{array}$} \\
\hline & Tam. Part. & $\%<0.452$ & Desde & Hasta \\
\hline 0 & 162.5 & 0.17 & 0.375 & $1,255.5$ \\
\hline 10 & 225.7 & 0.24 & 0.375 & $1,377.2$ \\
\hline 25 & 137.9 & 0.15 & 0.412 & 786.9 \\
\hline 35 & 129.3 & 0.23 & 0.375 & 741.8 \\
\hline 50 & 130.7 & 0.21 & 0.375 & 909.6 \\
\hline 70 & 134.8 & 0.15 & 0.375 & $1,094.2$ \\
\hline 85 & 160.5 & 0.24 & 0.375 & 111.0 \\
\hline
\end{tabular}




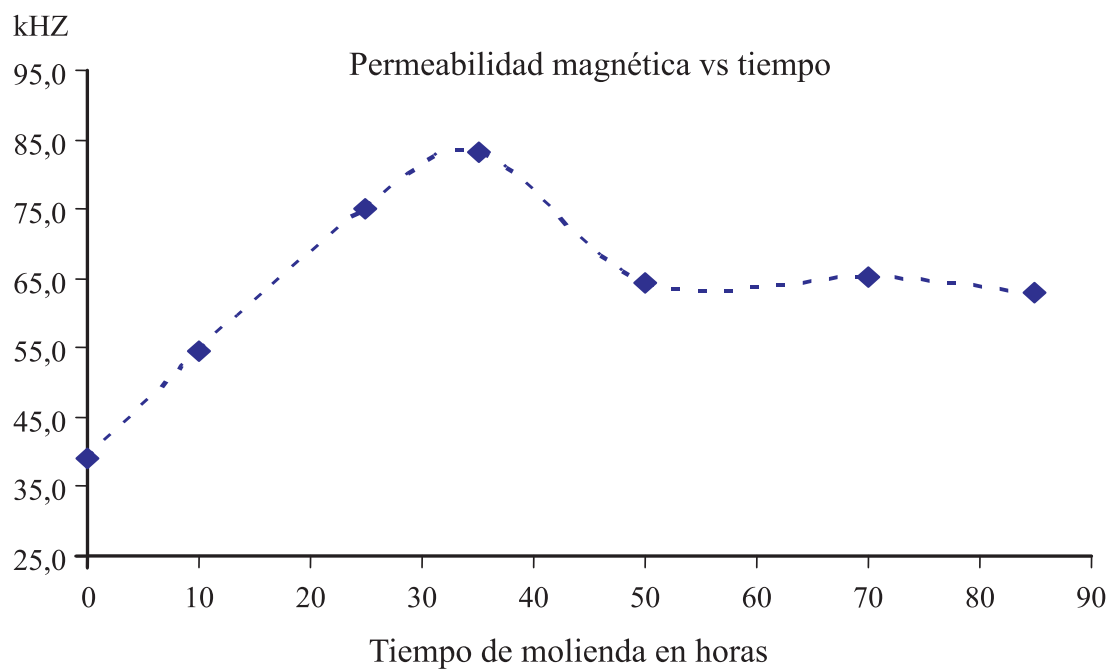

Figura 4. Variación de la permeabilidad magnética de las mezclas contra tiempo de molienda.

cuantitativo de fases aplicando el método de Rietveld. La producción máxima de ferrita estequiométrica de manganeso (cerca de $80 \mathrm{~mol} \%$ ) se obtuvo después de $35 \mathrm{~h}$ de molienda, considerándose este tiempo como el de óptima eficiencia alcanzada. A este mismo tiempo se alcanza la máxima permeabilidad magnética de este sólido, así como el menor tamaño promedio de partícula y la mayor homogeneidad. La contaminación por hierro proveniente de las herramientas de acero inoxidable (bolas e interior del frasco de molienda) no puede ser ignorada, ya que propicia la aparición de Fe metálico como fase no deseada. Debido a la contaminación por hierro, la ferrita sintetizada se descompone, dando como resultado una fase de mangano-wustita $\left(\mathrm{Fe}_{1-\mathrm{x}} \mathrm{Mn}_{\mathrm{x}} \mathrm{O}\right)$ de $\mathrm{Fe}(\mathrm{II})$ y $\mathrm{Mn}(\mathrm{II})$.

\section{Agradecimientos}

Este estudio fue financiado por el sistema del PII, soporte financiero de la Universidad Autónoma del Estado de Hidalgo, bajo el proyecto clave PAI06/01.

\section{Referencias bibliográficas}

Albani, C., G. Ennas, A. La Barbera, G. Marongiu, F. Padella, F. Varsano, 2005, Synthesis of nanocrystalline MnFe2O4: advances in thermochemical water splitting: International Journal Hydrogen Energy, 30 (13), p.1407-1411.

Beckman Coulter, 2001, Manual de Procedimiento del Equipo, Analizador de Tamaño de Partículas por Rayos Laser LS13-320, USA p. $1-11$.

Bergey, E. J., L. Levy, X. Wang, L.J. Krebs, M. Lal, K. Kim, S. Pakatchi, C. Liebow, P.N. Prasad, 2002, DC Magnetic Field Induced Magnetocytolysis of Cancer Cells Targeted by LH-RH Magnetic Nanoparticles in vitro: Biomedical Microdevices 4, 293-299.

Bid, S., S.K. Pradhan, 2003, Preparation of zinc ferrite by high-energy ball-milling and microstructure characterization by Rietveld's analysis: Materials Chemistry and Physics, 82(1), 27-37.
Bid, S., S.K. Pradhan, 2004, Characterization of crystalline structure of ball-milled nano-Ni-Zn-ferrite by Rietveld method: Materials Chemistry and Physics, 84(2), 291-301.

Bonsdorf, G., K. Schâfer, K. Teske, H. Langbein, H. Ullmann, 1998, Stability region and oxygen stoichiometry of manganese ferrite: Solid State Ionics, 110, 73-82.

Bremer, M., S. Fischer, H. Langbein, W. Topelmann, H. Scheler, 1992 Investigation on the formation of manganese-zinc ferrites by thermal decomposition of solid solution oxalates: Thermochimica Acta, 209, 323-330.

Carpenter, E., C.J. O'Connor, V.G. Harris, 1999, Atomic structure and magnetic properties of $\mathrm{MnFe} 2 \mathrm{O} 4$ nanoparticles produced by reverse micelle synthesis: Journal of Applied Physics, 85, 5175-5178.

Gajbhiye, N., G. Balaji, 2002, Synthesis, reactivity, and cations inversion studies of nanocrystalline $\mathrm{MnFe} 2 \mathrm{O} 4$ particles: Thermochimica Acta, 385 (1), 143-151.

Guillot, B., M. Laarj, S. Kacim, 1997, Reactivity towards oxygen and cation distribution of manganese iron spinel Mn3-xFexO4 $(0 \leq x \leq 3)$ fine powders studied by thermogravimetry and IR spectroscopy: Journal of Materials Chemistry, 7, 827-832.

Kaneko, H., Y. Ochiai, K. Shimizu, Y. Yosokawa, N. Gokon, Y. Tamaura, 2002, Thermodynamic study based on the phase diagram of the $\mathrm{Na} 2 \mathrm{O}-\mathrm{MnO}-\mathrm{Fe} 2 \mathrm{O} 3$ system for $\mathrm{H} 2$ production in three-step water: Solar Energy, 72 (4), 377-383.

Fachinformationszentrum (FIZ) Karlsruhe, 1999, Inorganic Crystal Structure Database, (en línea): Karlsruhe, Germany, base de datos y programa informático, consulta 13 de septiembre de 2007.

Kodama, T., M. Ookubo, S. Miura, Y. Kitayama, 1996, Synthesis and characterization of ultrafine $\mathrm{Mn}$ (II)-bearing ferrite of type $\mathrm{MnxFe} 3-\mathrm{xO} 4$ by coprecipitation: Materials Research Bulletin, 31(12), 1501-1512.

Liu, C., B. Zou, J. Rondinone, Z.J. Zhang, 2000, Reverse Micelle Synthesis and Characterization of Superparamagnetic MnFe2O4 Spinel Ferrite Nanocrystallites: Jorunal Physics Chemistry B, 104 (6), 1141-1145

Lutterotti, L. S.; Matthies, H.; Wenk, R., 2003, MAUD (Material Analysis Using Diffraction): a user friendly Java program for Rietveld Texture Analysis and more (en línea), in Proceeding of the Twelfth International Conference on Textures of Materials (ICOTOM-12), Vol. 1, 1999, Version 1.993 (programa informático), <<http://www. ing.unitn.it/luttero/maud. $>>$, consulta: 12 de noviembre de 2006.

Madsen, I., N.V.Y. Scarlett, L.M.D. Cranswick, T. Lwin, 2001, Outcomes of the International Union of Crystallography Commission on Powder Diffraction Round Robin on Quantitative Phase Analysis: 
samples 1a to 1h: Journal of Applied Crystallography, 34, 409426.

Magini, M., N. Burgio, A. Iasonna, S. Martelli, F. Padella, E. Paradiso, 1993, Estimation of entrapped powder temperature during mechanical alloying: Journal of Materials Synthesis and Processing, $1,135-141$.

Magini, M., A. Iasonna, 1995, Energy Transfer in Mechanical Alloying: Materials Transaction, JIM, Japan Institute of Metals, 36 (2), 123-133.

Maurice, D., T.H. Courtney, 1990, Milling and mechanical alloying of inorganic nonmetallics: Metallurgical and Materials Transactions A, 21(7), 1519-1525.

Rietveld, H, 1967, Line profiles of neutron powder-diffraction peaks for structure refinement: Acta Crystallographica, 22, 151-155.

Saferikova, M., L. Safarik, 2001, The application of magnetic techniques in biosciences: Magnetic and Electrical Separation, 10(2), 223252.

Sun, S., H. Zeng, B. Robinson, S. Raoux, P.M. Rice, S.X. Wang, G. Li., 2004, Shape-Controlled Synthesis and Shape-Induced Texture of $\mathrm{MnFe} 2 \mathrm{O} 4$ Nanoparticles: Journal of the American Chemical Society, 126(37), 11458-11459.

Tamaura, Y., Ueda, J. Matsunami, N. Hasegawa, M. Nezuka, T. Sano, M. Tsuji, 1999, Solar Hydrogen Production by Using Ferrites: Solar Energy, 65(1), 55-60.
Tamaura, Y., S. Steinfeld, P. Kuhn, K. Ehrensberger, 1995, On the oxygen-releasing step in the water-splitting thermochemical cycle by $\mathrm{MnFe} 2 \mathrm{O} 4-\mathrm{Na} 2 \mathrm{CO} 3$ system: Energy, 20(2), 325-330.

Tamaura, Y., M. Kojima, T. Sano, Y. Ueda, N. Hasegawa, M. Tsuji, 1998, Thermodynamic evaluation of water splitting by a cation-excessive (Ni, Mn) ferrite: International Journal Hydrogen Energy 23(12), $1185-1191$.

Young, R., 1993, The Rietveld Method, In: Ed. Intitute Union of Crystalographyc, Chapter 2, The Early Days: a Retrospective View, Oxford, University Press, New York.

Manuscrito recibido: Marzo, 9, 2007

Manuscrito corregido recibido: Marzo 31, 2007

Manuscrito aceptado: Abril 16, 2007 